\title{
Properties of recoverable region and semi-global stabilization in recoverable region for linear systems subject to constraints
}

\author{
Anton A. Stoorvogel *
}

\author{
Ali Saberi ${ }^{\dagger}$
}

\author{
Guoyong Shi *
}

\begin{abstract}
This paper investigates time-invariant linear systems subject to input and state constraints. It is shown that the recoverable region (which is the largest domain of attraction that is theoretically achievable) can be semiglobally stabilized by continuous nonlinear feedbacks while satisfying the constraints. Moreover, a reduction technique is presented which shows, when trying to compute the recoverable region, that we only need to compute the recoverable region for a system of lower dimension which generally leads to a considerable simplification in the computational effort.
\end{abstract}

\section{Introduction}

In this paper we revisit the problem of stabilization of general linear time-invariant systems subject to input and state constraints. Over the past years there has been rather strong interest in this problem, possibly due to a wide recognition of the inherent constraints on the input and state in most practical control systems. Consequently, several important results have appeared in the open literature. A result due to Sontag and Sussmann [21] shows that, for linear stabilizable systems, only systems which have no open-loop poles with positive real parts can be globally asymptotically stabilized by a bounded feedback. However, global stabilization in general requires a nonlinear controller as was established first by Fuller [9] and more recently by Sussmann and Yang [24]. Later, it is shown in Lin and Saberi [14] that systems which are globally stabilizable by nonlinear control laws are always semi-globally stabilizable by linear control laws. It is obvious that for systems having open-loop poles with positive real parts, global or semi-global stabilization with constrained input is impossible. The set of initial states that is stabilizable is normally a proper subset of the entire state space, i.e. the maximum achievable domain of attraction is a proper subset of the entire state space.

More recently, the global and semi-global stabilization results for input constraints are extended to linear systems with state and input constraints in [17], where global and semiglobal stabilization are defined relative to the admissible set. The admissible set is defined as the set of initial conditions that do not violate the constraints at time 0. Solvability conditions for global and semi-global stabilization with re- spect to the admissible set have been developed. It turns out that invariant zeros, infinite zeros and right-invertibility properties of the subsystem from control input to the constrained output play a crucial role. In [17] these invariant zeroes and infinite zeros are labeled as constraint invariant zeros and constraint infinite zeros. More specifically, the taxonomy of constraints presented in [17] delineates the constraints into several categories, such as right and non-right invertible constraints, minimum phase, at most weakly nonminimum phase, strongly non-minimum phase constraints, and type one constraints, etc. For systems with right invertible constraints, it is shown that the necessary conditions for global and semi-global stabilization are that the system is stabilizable and the constraints are at most weakly nonminimum phase (i.e. the constrained invariant zeros are in the closed left-half plane). Moreover for global stabilization one needs an additional condition that the constraints be of type one. For constraints that are right invertible and at most weakly non-minimum phase, it is possible to achieve semiglobal stabilization by a linear control; however, in general one has to use nonlinear control laws for global stabilization [17]. For the case of non-right invertible constraints, the situation is more complicated. The case of semi-global stabilization has recently been resolved in [19]. For global stabilization and output regulation only partial results are available and it remains a very complex and challenging problem that is yet to be resolved. If a system has at least one of the constraint invariant zeros in the open right-half plane, the so called non-minimum phase constraint, then neither semiglobal nor global stabilization in the admissible set is possible. In other words, the stabilization is possible only for some proper subset of the admissible set.

The notion of recoverable region (set), sometimes called domain of null controllability or null controllable region, is closely related to the stabilization of linear systems subject to constraints. Generally speaking, for a system with constraints, an initial state is said to be recoverable if it can be driven to zero by some control without violating the constraints on the state and input. The set of all recoverable initial conditions denoted by $\mathcal{R}_{C}$ is said to be the recoverable region. The recoverable region is closely related to the stabilization problem in the presence of non-minimum phase constraints for it represents the maximum achievable domain

\footnotetext{
*Dept. of Math. and Comp. Science, Eindhoven Univ. of Technology and Dept. of Inf. Techn. and Systems, Delft Univ. of Technology, The Netherlands

${ }^{\dagger}$ School of Electrical Engineering and Computer Science, Washington State University, Pullman, WA 99164-2752, U.S.A. E-mail: saberi@eecs.wsu.edu.

Electrical Engineering Department, University of Washington, Seattle, WA 98195, U.S.A. E-mail: gshi@ee.washington.edu
} 
of attraction. As such, the goal of stabilization is to design a feedback, say $u=f(x)$, such that the constraints are not violated and moreover the region of attraction of the equilibrium point of the closed loop system, say $\mathcal{R}_{A}$, is equal to the recoverable region, say $\mathcal{R}_{C}$, or is a large subset contained in $\mathcal{R}_{C}$. The earliest literature in this respect can be traced back to 1960's. For the case of input constraints, J. L. LeMay in 1964 first studied the conditions for characterizing the maximal region of recoverability and the maximal region of reachability [13]. LeMay also derived a method for calculation of recoverable regions based on optimal control techniques. It is known that for any state in the recoverable region there exists a time-optimal control law that drives the state to zero. This fact builds a direct connection between the characterization of the recoverable region and time-optimal control. There exists a vast literature in the 60's and 70's that were devoted to time-optimal control, among them we mention [8, 10, 12, 15]. E. P. Ryan [16] presented a set of very detailed results of time-optimal control of systems with input constraints whose number of unstable eigenvalues is between one and four. Note that in the case of only input constraints the recoverable region is completely determined by the unstable dynamics. More recently in 1995, Stephan et al. extended some of LeMay's results to systems with input and state constraints [22,23]. They examined computational issues of the recoverable regions for planar systems with state and input constraints.

There are two lines of research in the literature on stabilization problems in the presence of non-minimum phase constraints. A traditional line employs the construction of invariant sets $[1-4,7,26]$. The second line of research takes a fundamental view of global and semi-global stabilization relative to the recoverable region $[5,6,11,17]$. In this paper we focus on two issues. The first issue is properties and computational issues for the recoverable region. Our goal here is to provide a reduction in computation and removal of some of the computational complexity involved in obtaining the recoverable set. The second issue is semi-global stabilization via continuous state feedbacks in the recoverable region. In the special case that the constraints are right invertible, these questions were partially addressed in [17].

This paper is organized as follows. After the introduction we present some preliminary results in section 2 . In section 3 we discuss the issues related to computing the recoverable region and present a reduction technique which allows us to reduce the computational effort by developing an explicit relationship between the recoverable region of the full system and the recoverable region of a subsystem of lower order. In section 4 we establish that for any compact set contained in the interior of the recoverable region, there exists a continuous controller that stabilizes the system and contains the chosen compact set in its domain of attraction while satisfying the constraints. Due to page limitation, all proofs are omitted.

Notation: For a matrix $A, \operatorname{im} A$ denotes the image space of matrix $A$. For any set $\mathcal{C} \subset \mathbb{R}^{n}$, int $\mathcal{C}$ denotes the interior of set $\mathcal{C}, \partial \mathcal{C}$ the boundary of set $\mathcal{C}$, and $\overline{\mathcal{C}}$ the closure of set $\mathcal{C}$.

\section{Preliminaries}

This section provides the fundamentals for our development. We start with a description of our system model and its constraint. Then we introduce some basic notions that we are interested in. After that we recall a taxonomy of constraints related to the constrained system $\Sigma$ in (2.1). This taxonomy provides us basic terminologies for rest of the paper.

Consider the time-invariant linear system:

$$
\Sigma:\left\{\begin{array}{l}
\dot{x}=A x+B u \\
z=C_{z} x+D_{z} u
\end{array}\right.
$$

where $x \in \mathbb{R}^{n}$ is the state, $u \in \mathbb{R}^{m}$ is the control input, and $z \in \mathbb{R}^{p}$ is the constrained output, with the output $z \in \mathbb{R}^{p}$ subject to the constraint $z(t) \in \delta$ for all $t \geqslant 0$ where $\delta$ is a given subset of $\mathbb{R}^{p}$. Note that the case of input constraint is included as a special case in this general setup by letting $C_{z}=0$ and $D_{z}=I$ in the constrained output equation. However, one should note the difference of input saturation from the input constraint; a saturation can be overloaded, whereas a constraint can never be violated.

We make a general assumption on the constraint set $\delta$ and the structure of the constrained output.

Assumption 2.1 The set $\&$ is compact, convex and contains 0 as an interior point. Moreover, we assume $C_{z}^{T} D_{z}=0$ and

$$
\delta=\left(\delta \cap \operatorname{im} C_{z}\right)+\left(\delta \cap \operatorname{im} D_{z}\right)
$$

This assumption is not restrictive. In fact, it is a general reflection of the separability of input constraints and state constraints. Given the constraint on the output, obviously the initial states of the system must be restricted, since, if the initial state of the system is arbitrary then constraint violation can never be avoided. For this reason, we need to define an admissible set of initial conditions. It is straightforward to see that if an initial state is not in this set, then no controller can avoid constraint violation.

Definition 2.2 Given the system $\Sigma$ in (2.1) and a constraint set \& satisfying Assumption 2.1, the set $\mathcal{A}(\Sigma, \&) \subset \mathbb{R}^{n}$

$$
\mathcal{A}(\Sigma, 8):=\left\{x \in \mathbb{R}^{n} \mid C_{z} x \in \delta\right\}
$$

is said to be the admissible set of initial conditions.

Definition 2.3 Given system $\Sigma$ in (2.1) together with a constraint set $\&$ satisfying Assumption 2.1. The recoverable region $\mathcal{R}_{C}(\Sigma, 8)$ of this system is the set of all initial states $x(0) \in \mathcal{A}(\Sigma, 8)$ for which there exists a control input $u$ such that $x(t) \rightarrow 0$ as $t \rightarrow \infty$ while $z(t) \in \delta$ for all $t \geqslant 0$. 


\subsection{Taxonomy of Constraints}

We review briefly the taxonomy of constraints for the system $\Sigma$, given by (2.1), which has emerged from the study of stabilization of such systems [17]. It is known that certain structural properties of this system play important roles in the solvability of certain constrained stabilization problems. Specifically, right invertibility, the location of invariant zeros, and the order of infinite zeros of the quadruple ( $A, B, C_{z}, D_{z}$ ) dictate the solvability conditions for some constrained stabilization problems. The taxonomy of the constraints is based on these structural properties.

The first category in the taxonomy of constraints is based on whether the system $\Sigma$ is right invertible or not.

Definition 2.4 The constraints are said to be

- right invertible constraints if the system $\Sigma$ is right invertible.

- non-right invertible constraints if the system $\Sigma$ is nonright invertible.

The second category of constraints is based on the location of the invariant zeros of the system $\Sigma$, which are labeled the constraint invariant zeros of the plant. In the following definition, $\mathbb{C}^{-}, \mathbb{C}^{0}$, and $\mathbb{C}^{+}$denote respectively the set of complex numbers with negative real part, zero real part, and positive real part.

Definition 2.5 The constraints are said to be

- minimum phase constraints if all the constraint invariant zeros are in $\mathbb{C}^{-}$.

- at most weakly non-minimum phase constraints if all the constraint invariant zeros are in $\mathbb{C}^{-} \cup \mathbb{C}^{0}$.

- strongly non-minimum phase constraints if one or more of the constraint invariant zeros are in $\mathbb{C}^{+}$.

The third categorization is based on the order of the infinite zeros of the system $\Sigma$, which are labeled as the constraint infinite zeros of the plant.

Definition 2.6 The constraints are said to be type one constraints if the order of all constraint infinite zeros is less than or equal to one.

\section{The recoverable region}

This section is devoted to some characterizations of the recoverable region $\mathcal{R}_{C}(\Sigma, \S)$ of system $\Sigma$ as defined in Definition 2.3. The first set of properties of the recoverable region $\mathcal{R}_{C}(\Sigma, 8)$ are more or less well known. They are compiled in the following lemma for easy reference.

Lemma 3.1 Consider system $\Sigma$ in (2.1) and a compact, convex constraint set 8 containing 0 in the interior. The recoverable region $\mathcal{R}_{C}(\Sigma, 8)$ for this system has the following properties:

(i) If $(A, B)$ is controllable, then for any initial $x(0) \in$ $\mathcal{R}_{C}(\Sigma, 8)$ there exists $T>0$ and an input signal $u$ such that $x(T)=0$ while $z(t) \in \&$ for all $t \in[0, T]$. (ii) The set $\mathcal{R}_{C}(\Sigma, 8)$ is convex and contains the origin as an interior point.

(iii) If $(A, B)$ is stabilizable, then the set $\mathcal{R}_{C}(\Sigma, 8)$ is open in case we have only input constraints, i.e. $C_{z}=0$, but in general this need not be true.

(iv) The set $\mathcal{R}_{C}(\Sigma, 8)$ is bounded if all the invariant zeros of the system (2.1) are antistable and the system is left invertible with relative degree at most one.

Remark 3.1 Note that item (i) of the above lemma states that infinite-time recoverability is equivalent to finite-time recoverability.

Remark 3.2 Assume that $C_{z}=0$ and $D_{z}=I$ in (2.1), i.e. the system is only subject to input constraints and without state constraints. Then, under a suitable coordinate system in the state space, the plant can be split into two subsystems:

$$
\Sigma_{s}: \dot{x}_{s}=A_{s} x_{s}+B_{s} u, \quad \Sigma_{u}: \quad \dot{x}_{u}=A_{u} x_{u}+B_{u} u,
$$

where the eigenvalues of $A_{s}$ are in the closed left half plane (at most critically unstable) and those of $A_{u}$ are in the open right half plane (antistable). Then,

(i) $\mathcal{R}_{C}(\Sigma, \S)$ is an open set, and $\mathcal{R}_{C}\left(\Sigma_{u}, \S\right)$ is bounded;

(ii) $x \in \mathcal{R}_{C}(\Sigma, 8)$ if and only if $x_{u} \in \mathcal{R}_{C}\left(\Sigma_{u}, 8\right)$.

Note that when the system has state constraints, the admissible set need not be open. The fact stated above tells us that, without state constraints, the recoverable region is completely determined by the exponentially unstable part of the system. Because of the computational complexities involved in the characterization of the recoverable region, any reduction in dimension is beneficial from a computational point of view. On the other hand. for the case of state constraints this decomposition is no longer possible. Later in this section we show that in general a different type of order reduction is possible of which the above is actually a special case.

Next we present our first reduction result for the set $\mathcal{R}_{C}(\Sigma, \S)$. In order to do so first we represent $\Sigma$ in a special coordinate basis (scb). A brief review of scb is presented in Appendix A.

We can extract a subsystem from the full system in scb consisting of the state variables $x_{a}$ and $x_{b}$, input variable $\zeta$ consisting of $z_{0}$ and $z_{d}$ and output $\bar{z}$ :

$$
\Sigma_{1}:\left\{\begin{array}{l}
\dot{x}_{a}=A_{a} x_{a}+K_{a b} C_{b} x_{b}+K_{a 2} \zeta \\
\dot{x}_{b}=\left(A_{b}+K_{b b} C_{b}\right) x_{b}+K_{b 2} \zeta \\
\bar{z}=\left(\begin{array}{c}
C_{b} \\
0
\end{array}\right) x_{b}+\left(\begin{array}{c}
0 \\
I
\end{array}\right) \zeta .
\end{array}\right.
$$

Let $n_{1}=n_{a}+n_{b}$ be the state dimension of this system. Obviously $\zeta$ is not an input for the original system. However, for the moment we view $\zeta$ as the input to this subsystem while $\bar{z}$ is a constrained output for this subsystem. When transforming the system into scb, this clearly affects the constraint set 
and we obtain a new constraint set $\bar{s}=T_{z}^{-1} \&$. Thus, the constraint on $\bar{z}$ becomes

$$
\bar{z}(t) \in \bar{s}, \text { for all } t \geqslant 0,
$$

which is the constraint set for $\Sigma_{1}$. Let $\mathcal{R}_{C}\left(\Sigma_{1}, \bar{\delta}\right)$ be the recoverable region of subsystem $\Sigma_{1}$ with the constraint set $\bar{s}$.

The following theorem shows the relationship between the recoverable region of the full system $\Sigma$ and the recoverable region of the subsystem $\Sigma_{1}$.

Theorem 3.2 Consider the plant $\Sigma$ as given by (2.1) and $a$ constraint set $\&$ satisfying Assumption 2.1. Assume that we have extracted the subsystem $\Sigma_{1}$ in (3.1) from $\Sigma$ as described above. Then we have:

$$
\overline{\mathcal{R}_{C}(\Sigma, \S)}=\overline{T_{x}\left(\mathcal{R}_{C}\left(\Sigma_{1}, \bar{\jmath}\right) \times \mathbb{R}^{n-n_{1}}\right) \cap \mathcal{A}(\Sigma, \S)}
$$

Remark 3.3 The decomposition of the recoverable region $\mathcal{R}_{C}(\Sigma, 8)$ as presented in Theorem 3.2 is very important from a computational point of view. As pointed out in [23], numerical computation of recoverable regions suffers from dimension growth. In this sense, any reduction of dimension in the computation of the recoverable region is crucial for improvement of computation efficiency. Note that we have an explicit expression for $\mathcal{A}(\Sigma, 8)$ and therefore the above really reduces the computational question to determining $\mathcal{R}_{C}\left(\Sigma_{1}, \bar{s}\right)$.

Let us next have a another look at computational reduction in the system $\Sigma$. This different view of computational reduction will provide some interesting results for special cases. To do so we need to define another subsystem. Consider the remaining dynamics in the system $\Sigma$ besides the subsystem $\Sigma_{1}$. We consider the system in the special coordinate basis and, additionally, we define the new variables:

$$
\begin{aligned}
& \tilde{u}_{c}=u_{c}+H_{a} x_{a}, \\
& \tilde{u}_{d}=u_{d}+G_{a} x_{a}+G_{b} x_{b}+G_{c} x_{c}+G_{d} x_{d} .
\end{aligned}
$$

We also decompose $K_{c}$ and $K_{d}$ :

$$
\begin{aligned}
& K_{c}=\left(\begin{array}{lll}
K_{c b} & K_{c 0} & K_{c d}
\end{array}\right)=\left(\begin{array}{lll}
K_{c b} & K_{c 2}
\end{array}\right) \\
& K_{d}=\left(\begin{array}{lll}
K_{d b} & K_{d 0} & K_{d d}
\end{array}\right)=\left(\begin{array}{ll}
K_{d b} & K_{d 2}
\end{array}\right) .
\end{aligned}
$$

and we get the following description for the dynamics which together with $\Sigma_{1}$ describe the full system:

$$
\Sigma_{2}\left\{\begin{aligned}
\dot{x}_{c} & =A_{c} x_{c}+K_{c d} C_{d} x_{d}+K_{c 0} u_{0}+B_{c} \tilde{u}_{c}+K_{c b} z_{b} \\
\dot{x}_{d} & =\left(A_{d}+K_{d d} C_{d}\right) x_{d}+K_{d 0} u_{0}+B_{d} \tilde{u}_{d}+K_{d b} z_{b} \\
\zeta & =\left(\begin{array}{c}
0 \\
C_{d}
\end{array}\right) x_{d}+\left(\begin{array}{l}
I \\
0
\end{array}\right) u_{0}
\end{aligned}\right.
$$

Note that $\Sigma_{2}$ is only affected from $\Sigma_{1}$ via the signal $z_{b}$. We can set $z_{b}=0$ then we decouple $\Sigma_{2}$ from $\Sigma_{1}$ and when we also ignore the constraints on $z_{b}$ by setting

$$
\bar{s}_{2}:=\left\{\zeta \in \mathbb{R}^{n_{2}} \mid \exists z_{b} \text { such that }\left(z_{b}^{\mathrm{T}} \quad \zeta^{\mathrm{T}}\right)^{\mathrm{T}} \in \mathcal{\&}\right\},
$$

and view $\bar{\gamma}_{2}$ as the constraint set for $\Sigma_{2}$. In this way we define the recoverable region $\mathcal{R}_{C}\left(\Sigma_{2}, \bar{s}_{2}\right)$ for the second subsystem.

The following lemma establishes some connections between the recoverable region $\mathcal{R}_{C}(\Sigma, 8)$ of the original system and the recoverable regions of the two subsystems, i.e. $\mathcal{R}_{C}\left(\Sigma_{1}, \bar{\delta}\right)$ and $\mathcal{R}_{C}\left(\Sigma_{2}, \bar{\varsigma}_{2}\right)$ for special cases.

Theorem 3.3 Consider the plant $\Sigma$ as given by (2.1) and a constraint set $\&$ satisfying Assumption 2.1. Assume that system $\Sigma$ has been decomposed into two subsystems in scb as described by $\Sigma_{1}$ and $\Sigma_{2}$. Then we have the following properties:

(i) It holds that

$$
\begin{aligned}
& \mathcal{R}_{C}(\Sigma, \&) \subset T_{x}\left[\mathcal{R}_{C}\left(\Sigma_{1}, \bar{s}\right) \times \mathcal{R}_{C}\left(\Sigma_{2}, \bar{\varsigma}_{2}\right)\right], \\
& \text { and } \overline{\mathcal{R}_{C}\left(\Sigma_{2}, \bar{\jmath}_{2}\right)}=\mathcal{A}\left(\Sigma_{2}, \bar{s}\right) \text {. }
\end{aligned}
$$

(ii) If the constraint is right invertible, then $\bar{\gamma}_{2}=\bar{\S}$ and

$$
\overline{\mathcal{R}_{C}(\Sigma, 8)}=\overline{T_{x}\left[\mathcal{R}_{C}\left(\Sigma_{1}, \bar{\rho}\right) \times \mathcal{A}\left(\Sigma_{2}, \bar{\gamma}\right)\right]} .
$$

(iii) If the constraint is right invertible and of relative degree one, then $\mathcal{R}_{C}\left(\Sigma_{2}, \bar{\gamma}\right)=\mathcal{A}\left(\Sigma_{2}, \bar{\delta}\right)$ and

$$
\mathcal{R}_{C}(\Sigma, \diamond)=T_{x}\left[\mathcal{R}_{C}\left(\Sigma_{1}, \bar{s}\right) \times \mathcal{A}\left(\Sigma_{2}, \bar{s}_{2}\right)\right] .
$$

We have achieved a reduction from computing the recoverable region for the system $\Sigma$ to the computation of the recoverable region for the subsystem $\Sigma_{1}$. As noted before a reduction in system order is crucial in making the computation of the recoverable region feasible. The question remains whether we can achieve further reductions. In scb the matrix $A_{a}$ is in fact a block diagonal matrix (see Appendix A). With this one more step refining, subsystem $\Sigma_{1}$ becomes

$$
\Sigma_{1}:\left\{\begin{aligned}
\dot{x}_{a}^{-0} & =A_{a}^{-0} x_{a}^{-0}+K_{a b}^{-0} C_{b} x_{b}+K_{a 2}^{-0} \zeta \\
\dot{x}_{a}^{+} & =A_{a}^{+} x_{a}^{+}+K_{a b}^{+} C_{b} x_{b}+K_{a 2}^{+} \zeta \\
\dot{x}_{b} & =\left(A_{b}+K_{b b} C_{b}\right) x_{b}+K_{b 2} \zeta \\
\bar{z} & =\left(\begin{array}{c}
C_{b} \\
0
\end{array}\right) x_{b}+\left(\begin{array}{c}
0 \\
I
\end{array}\right) \zeta .
\end{aligned}\right.
$$

where $K_{a b}=\left(\begin{array}{c}K_{a b}^{-0} \\ K_{a b}^{+}\end{array}\right)$and $K_{a 2}=\left(\begin{array}{c}K_{a 2}^{-0} \\ K_{a 2}^{+}\end{array}\right)$.

Note that all eigenvalues of $A_{a}^{-0}$ are in the closed left-half plane, and all eigenvalues of $A_{a}^{+}$are in the open right-half plane.

We extract a subsystem from $\Sigma_{1}$ given by:

$$
\tilde{\Sigma}_{1}:\left\{\begin{aligned}
\dot{x}_{a}^{+} & =A_{a}^{+} x_{a}^{+}+K_{a b}^{+} C_{b} x_{b}+K_{a 2}^{+} \zeta, \\
\dot{x}_{b} & =\left(A_{b}+K_{b b} C_{b}\right) x_{b}+K_{b 2} \zeta \\
\bar{z} & =\left(\begin{array}{c}
C_{b} \\
0
\end{array}\right) x_{b}+\left(\begin{array}{l}
0 \\
I
\end{array}\right) \zeta .
\end{aligned}\right.
$$

with state dimension $n_{11}$. We can relate the recoverable region of $\Sigma_{1}$ to the recoverable region of $\tilde{\Sigma}_{1}$ and then, using theorem 3.2, we can relate the recoverable region of $\Sigma$ to the recoverable region of $\tilde{\Sigma}_{1}$ 
Theorem 3.4 Consider the plant $\Sigma$ as given by (2.1) and a constraint set $\&$ satisfying Assumption 2.1. Define $\Sigma_{1}$ by (3.1) and $\tilde{\Sigma}_{1}$ by (3.6). We have:

$$
\mathcal{R}_{C}\left(\Sigma_{1}, \bar{\S}\right)=\mathbb{R}^{n_{1}-n_{11}} \times \mathcal{R}_{C}\left(\tilde{\Sigma}_{1}, \bar{\S}\right) .
$$

and

$$
\overline{\mathcal{R}_{C}(\Sigma, \S)}=\overline{T_{x}\left(\mathcal{R}_{C}\left(\Sigma_{1}, \bar{\jmath}\right) \times \mathbb{R}^{n-n_{1}}\right) \cap \mathcal{A}(\Sigma, \S)}
$$

Note that the reduction of the computation of the recoverable region from $\Sigma$ to the computation of the recoverable region for the lower order system $\tilde{\Sigma}_{1}$ actually yields the result in remark 3.2 as a special case.

If the constraint is right invertible and at most weakly non-minimum phase then $\tilde{\Sigma}_{1}$ is actually an empty (zerodimensional) system and we have $\mathcal{R}_{C}\left(\Sigma_{1}, \bar{\delta}\right)=\mathbb{R}^{n_{a}}$. Therefore, we can explicitly characterize the recoverable region:

$$
\overline{\mathcal{R}_{C}(\Sigma, 8)}=\overline{T_{x}\left[\mathbb{R}^{n_{a}} \times \mathcal{A}\left(\Sigma_{2}, \bar{\gamma}_{2}\right)\right]} .
$$

Note that the work of E.P. Ryan in [16] makes it likely that if the system $\tilde{\Sigma}_{1}$ has order less than or equal to two then we have a reasonable chance to characterize the recoverable region more or less explicitly even though this has not been worked out yet for the case of state constraints. If the order of this subsystem is larger than two the computational effort to actually obtain the recoverable region will be high.

\section{Semi-global stabilization in the re- coverable region}

The first objective of this paper was the reduction in the computation of the recoverable region as outlined in the previous section. The second objective of this paper is to show the possibility of stabilization without violating the constraints for any compact subset $\mathcal{K}$ contained in the interior of $\mathcal{R}_{C}(\Sigma, \diamond)$ by a continuous feedback. Regarding the existence of continuous controllers, our main result is summarized in the following theorem.

Theorem 4.1 Given the linear time-invariant system $\Sigma$ in (2.1) with a constraint set \& satisfying Assumption 2.1. Assume that $(A, B)$ is stabilizable. Then, for any compact subset $\mathcal{K}$ contained in the interior of $\mathcal{R}_{C}(\Sigma, 8)$, there exists a continuous (in general nonlinear) feedback $u=f(x)$ such that the zero equilibrium point of the closed loop system is asymptotically stable with a domain of attraction containing $\mathcal{K}$ and moreover $z(t) \in \&$ for all $t \geqslant 0$ when $x(0) \in \mathcal{K}$.

\section{A A special coordinate basis}

We recall from $[18,20]$ special coordinate basis (scb) for system $\Sigma$ in (2.1). A system in scb reveals the inherent finite and infinite zero structures, which are crucial components in classifying the constraints and in facilitating the design.
For a general linear system $\Sigma$ in (2.1), one can choose appropriate coordinates in the state, input, and output spaces, say $x=T_{x} \bar{x}, u=T_{u} \bar{u}$, and $z=T_{z} \bar{z}$, where $T_{x}, T_{u}$, and $T_{z}$ are invertible matrices such that the system (2.1) takes the following form

$$
\bar{\Sigma}:\left\{\begin{array}{l}
\dot{x}_{a}=A_{a} x_{a}+K_{a} \bar{z} \\
\dot{x}_{b}=A_{b} x_{b}+K_{b} \bar{z} \\
\dot{x}_{c}=A_{c} x_{c}+B_{c}\left(u_{c}+H_{a} x_{a}\right)+K_{c} \bar{z}, \\
\dot{x}_{d}=A_{d} x_{d}+B_{d}\left(u_{d}+G_{a} x_{a}+G_{b} x_{b}+\right. \\
\left.G_{c} x_{c}+G_{d} x_{d}\right)+K_{d} \bar{z}, \\
\bar{z}=\left(\begin{array}{c}
z_{b} \\
z_{0} \\
z_{d}
\end{array}\right)=\left(\begin{array}{c}
C_{b} x_{b} \\
u_{0} \\
C_{d} x_{d}
\end{array}\right),
\end{array}\right.
$$

where $\bar{x}^{\mathrm{T}}=\left(\begin{array}{llll}x_{a}^{\mathrm{T}} & x_{b}^{\mathrm{T}} & x_{c}^{\mathrm{T}} & x_{d}^{\mathrm{T}}\end{array}\right)$ and $\bar{u}^{\mathrm{T}}=\left(\begin{array}{lll}u_{0}^{\mathrm{T}} & u_{c}^{\mathrm{T}} & u_{d}^{\mathrm{T}}\end{array}\right)$. Furthermore, the $x_{a}$ equation can be decomposed as

$$
\dot{x}_{a}^{-0}=A_{a}^{-0} x_{a}^{-0}+K_{a}^{-0} \bar{z}, \quad \dot{x}_{a}^{+}=A_{a}^{+} x_{a}^{+}+K_{a}^{+} \bar{z},
$$

where

$$
x_{a}=\left(\begin{array}{c}
x_{a}^{-0} \\
x_{a}^{+}
\end{array}\right), \quad A_{a}=\left(\begin{array}{cc}
A_{a}^{-0} & 0 \\
0 & A_{a}^{+}
\end{array}\right), \quad K_{a}=\left(\begin{array}{c}
K_{a}^{-0} \\
K_{a}^{+}
\end{array}\right),
$$

all eigenvalues of $A_{a}^{-0}$ are in the closed left-half plane, and all eigenvalues of $A_{a}^{+}$are in the open right-half plane.

The scb components have the following dimensions. For the state, $x_{a} \in \mathbb{R}^{n_{a}}, x_{b} \in \mathbb{R}^{n_{b}}, x_{c} \in \mathbb{R}^{n_{c}}$, and $x_{d} \in \mathbb{R}^{n_{d}}$, with $n_{a}+n_{b}+n_{c}+n_{d}=n . x_{a}^{-0} \in \mathbb{R}^{n_{a}^{-0}}, x_{a}^{+} \in \mathbb{R}^{n_{a}^{+}}$, and $n_{a}^{-0}+n_{a}^{+}=n_{a}$. For the input, $u_{0} \in \mathbb{R}^{m_{0}}, u_{c} \in \mathbb{R}^{m_{c}}$, and $u_{d} \in \mathbb{R}^{\ell}$, with $m_{0}+m_{c}+\ell=m$. And for the output, $z_{0} \in \mathbb{R}^{m_{0}}, z_{b} \in \mathbb{R}^{m_{b}}$, and $z_{d} \in \mathbb{R}^{\ell}$, with $m_{0}+m_{b}+\ell=p$. Moreover, $A_{d}, B_{d}$, and $C_{d}$ have the following structure:

$$
\begin{aligned}
A_{d} & =\operatorname{block} \operatorname{diag}\left[A_{1}, \cdots, A_{\ell}\right], \\
B_{d} & =\operatorname{block} \operatorname{diag}\left[B_{1}, \cdots, B_{\ell}\right], \\
C_{d} & =\operatorname{block} \operatorname{diag}\left[C_{1}, \cdots, C_{\ell}\right],
\end{aligned}
$$

where $A_{i}=\left(0, e_{1}, \cdots, e_{k_{i}-1}\right) \in \mathbb{R}^{k_{i} \times k_{i}}, B_{i}=e_{k_{i}} \in$ $\mathbb{R}^{k_{i} \times 1}$, and $C_{i}=e_{1}^{\mathrm{T}} \in \mathbb{R}^{1 \times k_{i}}$ for $i=1, \cdots, \ell$, where $e_{i}$ is the $i$ th standard basis vector with appropriate dimension, and $\sum_{i=1}^{\ell} k_{i}=n_{d}$. According to the structure of $A_{d}$, the state $x_{d}$ is further decomposed to $x_{d}=\left(x_{1}^{\mathrm{T}}, \cdots, x_{\ell}\right)^{\mathrm{T}}$, where $x_{i} \in \mathbb{R}^{k_{i}}$, for $i=1, \cdots, \ell$.

The components involved in scb have lots of nice properties. Among others we mention the following that are relevant to this work:

(i) The eigenvalues of $A_{a}$ are the invariant zeros of the system $\Sigma$.

(ii) The infinite zeros are associated with the dynamics of $x_{d}$, and the order of infinite zero associated with $A_{i}$ is $k_{i}$. The relative degree of system $\Sigma$ refers to the greatest of $k_{i}, i=1, \cdots, \ell$.

(iii) The matrix pair $\left(A_{c}, B_{c}\right)$ is controllable. 
(iv) The matrix pair $\left(C_{b}, A_{b}\right)$ is observable.

(v) If system $\Sigma$ is right invertible, then the dimension of $x_{b}$ is zero. In this case, the components $x_{b}$ and $z_{b}$ disappear.

(vi) If system $\Sigma$ is left invertible, then the dimension of $x_{c}$ is zero. In this case, the components $x_{c}$ and $u_{c}$ disappear.

\section{Acknowledgement}

The research leading to the results presented in this paper was initiated by the first author in collaboration with his student Camiel van Moll. The results were described in the thesis [25] by Camiel van Moll and was for the case of input constraints only but raised and addressed many of the issues related to theorem 4.1 as described in this paper. The work of Camiel van Moll is gratefully acknowledged.

\section{References}

[1] G. Bitsoris, "On the linear decentralized constrained regulation problem of discrete-time dynamical systems", Information ansd Decision Technologies, 14(3), 1988, pp. 229-239.

[2] F. BlAnCHINI, "Set invariance in control", Automatica, 35(11), 1999, pp. 1747-1769.

[3] F. Blanchini and S. Miani, "Constrained stabilization of continuous-time linear systems", Syst. \& Contr. Letters, 28(2), 1996, pp. 95-102.

[4] _ "Constrained stabilization via smooth Lyapunov functions", Syst. \& Contr. Letters, 35, 1998, pp. 155163.

[5] J. CHOI, "On the stabilization of linear discrete-time systems subject to input saturation", Systems \& Control Letters, 36, 1999, pp. 241-244.

[6] _ , "On the constrained asymptotic stabilizability of unstable linear discrete time systems via linear feedback", in Proc. ACC, 2001, pp. 4926-4929.

[7] M. CWikel AND P. Gutman, "Convergence of an algorithm to find maximal state constraint sets for discrete-time linear dynamical systems with bounded controls and states", IEEE Trans. Aut. Contr., 31, 1986, pp. 457-459.

[8] I. FLÜGGE-Lotz, Discontinuous and optimal control, McGraw-Hill, New York, 1968.

[9] A.T. FulLER, "In-the-large stability of relay and saturating control systems with linear controller", Int. J. Contr., 10(4), 1969, pp. 457-480.

[10] - Ed., Nonlinear stochastic control systems, Taylor and Francis, London, 1970.

[11] T. HU, Z. LIN, AND L. QIU, "Stabilization of exponentially unstable linear systems with saturating actuators”, IEEE Trans. Aut. Contr., 45(6), 2001, pp. 973979.
[12] E.B. LEE AND L. MARKUS, Foundations of optimal control theory, John Wiley \& Sons, New York, 1967.

[13] J.L. LEMAY, "Recoverable and reachable zones for control systems with linear plants and bounded controller outputs", IEEE Trans. Aut. Contr., 9, 1964, pp. 346-354.

[14] Z. LiN AND A. SABERI, "Semi-global exponential stabilization of linear systems subject to "input saturation" via linear feedbacks", Syst. \& Contr. Letters, 21(3), 1993, pp. 225-239.

[15] L.S. Pontryagin, V.G. Boltyanskit, R.V. GAMKRELIDZE, AND E.R. MISCHENKO, The mathematical theory of optimal processes, Wiley, New York, 1962.

[16] E.P. RYAN, Optimal relay and saturating control system synthesis, Peter Peregrinus Ltd., 1982.

[17] A. Saberi, J. Han, And A.A. Stoorvogel, "Constrained stabilization problems for linear plants", Automatica, 38(4), 2002, pp. 639-654.

[18] A. SABERI AND P. SANnUti, "Squaring down of nonstrictly proper systems", Int. J. Contr., 51(3), 1990, pp. 621-629.

[19] A. Saberi, A.A. Stoorvogel, G. Shi, AND P. SANNUTI, "Semi-global stabilization of linear systems subject to non-right invertible constraints", Submitted for publication, a short version will appear in the proceedings of the American Control Conference, Denver, 2003.

[20] P. SANNUTI AND A. SABERI, "Special coordinate basis for multivariable linear systems - finite and infinite zero structure, squaring down and decoupling", Int. J. Contr., 45(5), 1987, pp. 1655-1704.

[21] E.D. Sontag AND H.J. Sussmann, "Nonlinear output feedback design for linear systems with saturating controls", in Proc. 29th CDC, Honolulu, 1990, pp. 3414-3416.

[22] J. Stephan, M. Bodson, And J. LehoczKy, "Properties of recoverable sets for systems with input and state constraints", in Proc. American Control Conf., 1995, pp. 3912-3913.

[23] — "Calculation of recoverable sets for systems with input and state constraints", Opt. Control Appl. \& Meth., 19, 1998, pp. 247-269.

[24] H.J. SuSSMANN AND Y. YANG, “On the stabilizability of multiple integrators by means of bounded feedback controls", Tech. Report SYCON-91-01, Rutgers Center for Systems and Control, 1991.

[25] C. VAN MOLL, "Stabilization of the null controllable region of linear systems with bounded continuous feedbacks.", master's thesis, Eindhoven University of Technology, 1999.

[26] M. VAssilaki, J.C. Hennet, And G. Bitsoris, "Feedback control of linear discrete-time systems under state and control constraints", Int. J. Contr., 47(6), 1988, pp. 1727-1735. 